\title{
Relaxation in BV for a class of functionals without continuity assumptions
}

\author{
M. AMAR and V. DE CICCO \\ Dipartimento di Metodi e Modelli Matematici \\ Università "La Sapienza" di Roma \\ Via A. Scarpa 16 \\ 00161 Roma, ITALY \\ e-mails: amar@dmmm.uniroma1.it \\ decicco@dmmm.uniroma1.it
}

\begin{abstract}
This paper is devoted to prove new relaxation and $\Gamma$-convergence theorems on $\mathrm{BV}(\Omega)$ for a class of integral functionals, whose integrands have a product type structure, but they do not satisfy any assumptions of coerciveness or continuity with respect to the spatial variable.
\end{abstract}

2000 Mathematics Subject Classification: 49J45, 49Q20, 49M20.

Key words: Relaxation, BV-functions, $\Gamma$-convergence.

\section{Introduction}

In this paper we study a relaxation problem in $\mathrm{BV}(\Omega)$ for integral functionals with linear growth which depend on scalar functions $u$. More precisely, we consider the following integral functional

$$
F(u)=\int_{\Omega} f(x, u(x), \nabla u(x)) d x, \quad u \in W^{1,1}(\Omega),
$$

and we study its $L^{1}$-lower semicontinuous extension to $\mathrm{BV}(\Omega)$ by using the relaxation method.

Our interest is to investigate the dependence of the integrand $f(x, s, \xi)$ with respect to the spatial variable $x$ and to consider discontinuous integrands too, in relation to the problem of finding an explicit representation of the relaxed functional.

Many authors contributed to the study of (1.1) with the aim to find the minimal assumptions on the integrand $f$ that guarantee its $L^{1}$-lower semicontinuity 
on $W^{1,1}(\Omega)$, and to obtain an integral representation for the relaxed functional of (1.1) on $\mathrm{BV}(\Omega)$.

For functionals of the type in (1.1), a classical result is the Serrin Theorem (see [33]), which establishes the lower semicontinuity of $(1.1)$ on the space $W^{1,1}(\Omega)$ with respect to the $L^{1}$-convergence by assuming that $f$ is continuous in all its variables, convex in the gradient variable and satisfying one of the following conditions: coerciveness; strict convexity in the last variable; continuity of the derivatives $f_{x}$, $f_{\xi}$ and $f_{x \xi}$.

After Serrin's Theorem, many authors generalized his result by weakening either the continuity assumption on $f$ or one of the above conditions. In this direction we recall a classical result due to De Giorgi, Buttazzo and Dal Maso (see [14]) in which the authors proved that the continuity of $f$ in $s$ is not necessary in order to obtain the $L^{1}$-lower semicontinuity of (1.1).

Recently, a new interest in this framework appeared, with the aim to weaken the continuity assumption on the variable $x$. In the last years, Gori, Maggi and Marcellini in [27] (see also [25]) proved the $L^{1}$-lower semicontinuity by requiring only a $W^{1,1}$-dependence of $f$ in $x$ (see also [13] for further improvements). In a more recent paper, Fusco, Gori and Maggi (see [22]) proved that the semicontinuity result under coerciveness or strict convexity hypothesis of Serrin's Theorem follows from the lower semicontinuity proved in the last part of the same theorem, under the regularity assumption. This surprising fact leads to investigate the behaviour of the gradient of $f$ with respect to $x$. In this direction, in [12] the authors proved the $\mathrm{L}^{1}$-lower semicontinuity of $(1.1)$ in $W^{1,1}(\Omega)$ by assuming only a $\mathrm{BV}$-dependence of $f$ with respect to the variable $x$.

Some of the previous lower semicontinuity results have been proven also for the functional

$$
\begin{aligned}
\mathcal{F}(u)= & \int_{\Omega} f(x, u, \nabla u) d x+\int_{\Omega} f^{\infty}\left(x, \widetilde{u}, D_{c} u\right) \\
& +\int_{S_{u} \cap \Omega}\left(\int_{u^{-}(x)}^{u^{+}(x)} f^{\infty}\left(x, s, \nu_{u}\right) d s\right) d \mathcal{H}^{N-1}(x),
\end{aligned}
$$

which is an extension of (1.1) to the larger space $\mathrm{BV}(\Omega)$ (see, for instance, [9] and [10]). Moreover, in [9] it is proved that, under continuity and coerciveness assumptions on the integrand, $\mathcal{F}$ coincides with the relaxed functional $\bar{F}$ of $F$ on $\mathrm{BV}(\Omega)$. Other representation results are given in [1] and [4].

Very recent developments on this subject are due to Fonseca and Leoni (see [19] and [20]). They improved the lower semicontinuity and relaxation theorem in [9], by omitting any coercivity assumptions and by requiring a continuous dependence of $f$ in $x$ (uniformly with respect to the other variables). On the other hand, in [26] and [28], the authors improved the results in [9], replacing the coercivity requirement with a weaker property, called demicoercivity, and assuming that $f$ is pointwise continuous with respect to all its variables.

In the last years, the lower semicontinuity result of Fonseca and Leoni was generalized firstly in [21] and later in [11]. In particular, in this last paper, the 
authors obtained the $L^{1}$-lower semicontinuity of $\mathcal{F}$ on $\mathrm{BV}(\Omega)$ assuming only a $W^{1,1}$-dependence of $f$ in $x$, in the spirit of the result in [27].

In the present paper, just moving from the result in [11], we will approach the relaxation problem under similar assumptions. Namely, we will prove the representation formula $\bar{F}=\mathcal{F}$, for integrands of the type

$$
f(x, s, \xi)=a(x, s) p(s, \xi)
$$

by assuming the $W^{1,1}$-dependence in $x$, the Lipschitz-continuity in $s$, the convexity and the linear growth in $\xi$ (see Theorem 3.8). To this aim, in the first part of the paper we will prove a continuity theorem for functionals defined on $\mathrm{BV}(\Omega)$ of this type

$$
G(v):=\int_{\Omega} A(z) P\left(\frac{D v}{|D v|}(z)\right) d|D v|(z)
$$

with respect to the strict convergence in $\mathrm{BV}(\Omega)$ (i.e. the $L^{1}$-convergence of the functions and the convergence of their total variations) under the assumptions that $A$ is a $W^{1,1}$-function and $P$ is convex, positively 1-homogeneous and with linear growth. In the proof of the relaxation result, this continuity criterion, which is actually stated in a more general form (see Theorem 3.3), plays the role of the classical Reshetnyak Continuity Theorem for functionals defined on measures, which holds only if the integrand is continuous (see Theorem 2.3). On the contrary, our result holds also for discontinuous integrands, but only for measures which are gradients of BV-functions. The proof of the continuity theorem is strongly based on the separation of the spatial variable and the gradient variable and it is an unexpected easy consequence of the lower semicontinuity theorem in [11] (see Remark 3.4).

We point out that our relaxation result produces a new class of functionals for which the formula $\bar{F}=\mathcal{F}$ holds, but the continuity of the integrand with respect to the spatial variable does not have necessarily to be assumed. We note that the condition imposed on the integrands (i.e., the separation of variables) is not so restrictive if we compare our result, for instance, with some examples given in [9, Section 4], where, for integrands with non separated variables, the relaxation formula does not hold.

Actually, the product type structure of the integrand will be removed in a forthcoming paper (see [2]), but to this aim we need a very deep analysis of the recession function, which is very delicate even in the separated case. On the contrary, the proof of the result we present here is obtained with a completely different approach in a surprisingly very simple way; but, unfortunately, this technique cannot be extended to the general case.

In the last part of the paper, we apply the previous relaxation theorem in order to prove a $\Gamma$-convergence result for a sequence of functionals whose integrands pointwise converge. Since we do not assume the continuity with respect to $x$ and the coerciveness condition, our result improves an analogous theorem proven in [9, Theorem 3.4], at least for functionals with integrand having product type structure. 
The paper is organized as follows: Section 2 is devoted to definitions and some preliminaries. In Section 3 we state our main results: firstly we prove the continuity theorem; then we establish the relaxation formula; finally, we establish the $\Gamma$-convergence result. In the last part, we give a counterexample which shows that, in order to obtain the Continuity Theorem 3.3 (and hence the relaxation result), it is necessary some control of the gradient of the integrand with respect to $x$.

\section{Notations and Preliminaries}

\subsection{Notations}

Throughout the paper, $N>1$ is a fixed integer.

Given $x_{0} \in \mathbb{R}^{N}$ and $\rho>0, B_{\rho}\left(x_{0}\right)$ denotes the ball in $\mathbb{R}^{N}$ centered in $x_{0}$ with radius $\rho$.

Let $\Omega$ be an open bounded subset of $\mathbb{R}^{N}$. We denote by $\mathcal{M}\left(\Omega ; \mathbb{R}^{N}\right)$ the space of the $\mathbb{R}^{N}$-valued finite Radon measures on $\Omega$; in particular, $\mathcal{M}(\Omega):=\mathcal{M}(\Omega ; \mathbb{R})$. Given a sequence of measures $\left\{\mu_{h}\right\} \in \mathcal{M}\left(\Omega ; \mathbb{R}^{N}\right)$, we say that $\left\{\mu_{h}\right\}$ strictly converges to $\mu \in \mathcal{M}\left(\Omega ; \mathbb{R}^{N}\right)$, if $\left\{\mu_{h}\right\}$ converges to $\mu$ weakly* in the sense of measures and $\left|\mu_{h}\right|(\Omega) \rightarrow|\mu|(\Omega)$.

Let $\mathcal{L}^{N}$ be the Lebesgue measure on $\mathbb{R}^{N}$, and $\mathcal{H}^{N-1}$ be the Hausdorff measure of dimension $(N-1)$ on $\mathbb{R}^{N}$. We denote by $\omega_{N}$ the Lebesgue measure of the unit ball in $\mathbb{R}^{N}$, so that $\mathcal{L}^{N}\left(B_{\rho}\left(x_{0}\right)\right)=\omega_{N} \rho^{N}$.

\subsection{Approximate continuity}

Let $u \in L_{\text {loc }}^{1}(\Omega)$; we say that $u$ has an approximate limit at $x \in \Omega$, if there exists a unique value $\widetilde{u}(x) \in \mathbb{R}$ such that

$$
\lim _{\varepsilon \rightarrow 0^{+}} f_{B_{\varepsilon}(x)}|u(y)-\widetilde{u}(x)| d x=0,
$$

where $f_{B_{\varepsilon}(x)}$ stands for $\frac{1}{\mathcal{L}^{N}\left(B_{\varepsilon}(x)\right)} \int_{B_{\varepsilon}(x)}$. Let $S_{u}$ be the set of points where the previous property does not hold, the so-called approximate discontinuity set. Note that $S_{u}$ is a Borel set and $\tilde{u}: \Omega \backslash S_{u} \rightarrow \mathbb{R}$ is a Borel function, called the precise representative of $u$. Clearly, if $x$ is a Lebesgue point of $u$, then (2.1) holds with $\widetilde{u}(x)$ replaced by $u(x)$. Moreover, we recall that $\mathcal{L}^{N}$-almost every $x \in \Omega$ is a Lebesgue point of $u$. Finally if, for some $x \in \Omega, u(x)=\widetilde{u}(x)$, we say that $u$ is approximately continuous at $x$.

\subsection{The space BV}

The space $\operatorname{BV}(\Omega)$ is defined as the space of those functions $u: \Omega \rightarrow \mathbb{R}$ belonging to $L^{1}(\Omega)$ whose distributional gradient $D u$ is an $\mathbb{R}^{N}$-valued Radon measure (i.e., $\left.D u \in \mathcal{M}\left(\Omega ; \mathbb{R}^{N}\right)\right)$ with total variation $|D u|$ bounded in $\Omega$. Given a sequence $\left\{u_{h}\right\}$ 
of BV-functions, we say that $\left\{u_{h}\right\}$ strictly converges to $u \in \mathrm{BV}(\Omega)$, if $u_{h} \rightarrow u$ strongly in $L^{1}(\Omega)$ and the total variations $\left|D u_{h}\right|(\Omega) \rightarrow|D u|(\Omega)$.

We indicate by $D_{a} u$ and $D_{s} u$ the absolutely continuous and the singular part of the measure $D u$ with respect to the Lebesgue measure. We recall that $D_{a} u$ and $D_{s} u$ are mutually singular, moreover we can write

$$
D u=D_{a} u+D_{s} u \quad \text { and } \quad D_{a} u=\nabla u d \mathcal{L}^{N},
$$

where $\nabla u$ is the Radon-Nikodým derivative of $D_{a} u$ with respect to the Lebesgue measure. In particular,

$$
D_{s} u=D_{c} u+\left(u^{+}-u^{-}\right) \nu_{u} d \mathcal{H}^{N-1}\left\lfloor S_{u}\right.
$$

where $S_{u}$ is defined above and it can be decomposed into two subsets $J_{u}$ and $S_{u} \backslash J_{u}$, with $\mathcal{H}^{N-1}\left(S_{u} \backslash J_{u}\right)=0$ and $J_{u}$, which is countably $\mathcal{H}^{N-1}$-rectifiable. The set $J_{u}$ is the so-called jump set of $u$, i.e. $x_{0} \in J_{u}$ if and only if there exists $u^{+}, u^{-} \in \mathbb{R}$ and $\nu_{u} \in S^{N-1}$ such that $u^{+}>u^{-}$and

$$
\lim _{\varepsilon \rightarrow 0^{+}} f_{B_{\varepsilon}^{ \pm}\left(x_{0}, \nu_{u}\right)}\left|u(x)-u^{ \pm}\right| d x=0,
$$

where $B_{\varepsilon}^{ \pm}\left(x_{0}, \nu_{u}\right)=x_{0}+\varepsilon B_{\nu_{u}}^{ \pm}$and $B_{\nu_{u}}^{ \pm}=\left\{x \in B_{1}(0):\left\langle x, \nu_{u}\right\rangle>0\right.$ (respect. $<0)\}$. The quantity $\left(u^{+}-u^{-}\right)$is the jump of $u$ across the interface $S_{u}$, in $\mathcal{H}^{N-1}$ almost every points; $\nu_{u}$ is the direction of the jump. Finally, $D_{c} u$ is the Cantor part of $D u$.

We define the subgraph of a given function $u: \Omega \rightarrow \mathbb{R}$ as the measurable subset of $\Omega \times \mathbb{R}$ defined by

$$
\mathcal{G}(u):=\{(x, t) \in \Omega \times \mathbb{R}: t<u(x)\} .
$$

We will denote by $\chi_{\mathcal{G}(u)}$ the characteristic function of $\mathcal{G}(u)$ and $\alpha(u)$ its distributional derivative. By a well known result of Miranda (see, for instance, [23, Chapter 4.1.5, Theorem 1]), it follows that, if $u \in \mathrm{BV}(\Omega)$, then $\mathcal{G}(u)$ is a set of finite perimeter in $\Omega \times \mathbb{R}$; i.e., $\chi_{\mathcal{G}(u)} \in \mathrm{BV}_{l o c}(\Omega \times \mathbb{R})$ and $\alpha(u) \in \mathcal{M}\left(\Omega \times \mathbb{R} ; \mathbb{R}^{N+1}\right)$. Moreover, the measure $\alpha(u)$ is concentrated over the reduced boundary of the subgraph of $u$ and the Radon-Nikodým derivative of $\alpha(u)$ with respect to its total variation is the inward normal to the subgraph of $u$.

For a general survey on measures and BV-functions we refer to $[3,17,18$, $24,29,32,34]$.

\subsection{Preliminary results}

Let $f: \Omega \times \mathbb{R} \times \mathbb{R}^{N} \rightarrow[0,+\infty)$ be a Borel function, convex with respect to the last variable. We define the so-called recession function of $f$ by

$$
\begin{aligned}
f^{\infty}(x, s, \xi) & =\lim _{t \rightarrow+\infty} \frac{f(x, s, t \xi)}{t} \\
& =\sup _{t>0} \frac{f(x, s, t \xi)-f(x, s, 0)}{t} \quad \forall(x, s, \xi) \in \Omega \times \mathbb{R} \times \mathbb{R}^{N} .
\end{aligned}
$$


We recall that the previous limit exists (since the function $t \mapsto \frac{f(x, s, t \xi)-f(x, s, 0)}{t}$ is increasing), the function $f^{\infty}$ is a Borel function, convex and positively 1-homogeneous in the last variable and, as a consequence of its definition, we have also that

$$
\frac{f(x, s, t \xi)}{t} \leq f^{\infty}(x, s, \xi)+\frac{f(x, s, 0)}{t} .
$$

Let us define $\widehat{f}: \Omega \times \mathbb{R} \times \mathbb{R}^{N} \times(-\infty, 0] \rightarrow[0,+\infty]$ as

$$
\widehat{f}(x, s, \xi, \tau):= \begin{cases}-f(x, s,-\xi / \tau) \tau & \text { if } \tau<0 \\ f^{\infty}(x, s, \xi) & \text { if } \tau=0 .\end{cases}
$$

Note that $\widehat{f}$ is a Borel function, convex and positively 1-homogeneous, with respect to the last two variables $(\xi, \tau)$.

For every Borel function $f$ as above, set $\mathcal{F}: \mathrm{BV}(\Omega) \rightarrow[0,+\infty]$ defined by

$$
\begin{aligned}
\mathcal{F}(u)= & \int_{\Omega} f(x, u, \nabla u) d x+\int_{\Omega} f^{\infty}\left(x, \widetilde{u}, D_{c} u\right) \\
& +\int_{S_{u} \cap \Omega}\left(\int_{u^{-}(x)}^{u^{+}(x)} f^{\infty}\left(x, s, \nu_{u}\right) d s\right) d \mathcal{H}^{N-1}(x),
\end{aligned}
$$

where, since $f^{\infty}(x, s, \cdot)$ is positively 1-homogeneous, $\int_{\Omega} f^{\infty}\left(x, \widetilde{u}, D_{c} u\right)$ stands for

$$
\int_{\Omega} f^{\infty}\left(x, \widetilde{u}(x), \frac{D_{c} u}{\left|D_{c} u\right|}(x)\right) d\left|D_{c} u\right|(x) .
$$

Moreover, if $f(x, s, \cdot)$ is positively 1-homogeneous, we have that $f=f^{\infty}$ and the functional in (2.5) can be written in an abbreviated form as

$$
\mathcal{F}(u)=\int_{\Omega}\left(f_{u^{-}(x)}^{u^{+}(x)} f\left(x, s, \frac{D u}{|D u|}(x)\right) d s\right) d|D u|(x),
$$

with the convention that if $g$ is a Borel function in $\mathbb{R}$ and $a \leq b$, then

$$
f_{a}^{b} g(s) d s=\left\{\begin{array}{lll}
\frac{1}{b-a} \int_{a}^{b} g(s) d s & \text { if } & a<b \\
g(a) & \text { if } & \mathrm{a}=\mathrm{b} .
\end{array}\right.
$$

The following lemma, which is proven in [9, Lemma 2.2] gives a representation formula for the functional $\mathcal{F}(u)$, in terms of an integral functional over the reduced boundary of the subgraph of $u$ in the space $\Omega \times \mathbb{R}$. This is one of the essential tools in order to obtain the relaxation result, which will be proven in Section 3.2.

Lemma 2.1 Assume that $f: \Omega \times \mathbb{R} \times \mathbb{R}^{N} \rightarrow[0,+\infty)$ is as before. Then,

$$
\mathcal{F}(u)=\int_{\Omega \times \mathbb{R}} \widehat{f}\left(x, s, \frac{\alpha(u)}{|\alpha(u)|}(x, s)\right) d|\alpha(u)|(x, s) \quad \forall u \in \operatorname{BV}(\Omega),
$$

where $\alpha(u)$ is the derivative of the characteristic function of the subgraph of $u$. 
Now we recall the $L^{1}$-lower semicontinuity result proven in [11].

Theorem 2.2 Assume that $f: \Omega \times \mathbb{R} \times \mathbb{R}^{N} \rightarrow[0,+\infty)$ is a Borel function, locally bounded, continuous in the second variable, convex in the last variable and satisfying the following conditions:

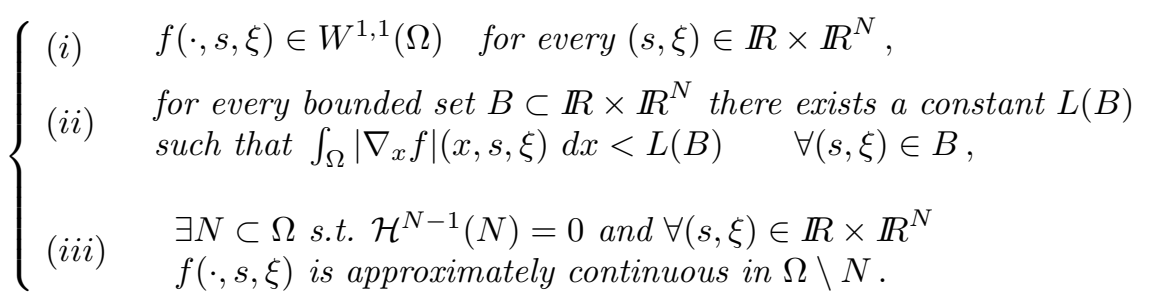

Then the functional $\mathcal{F}$ defined in (2.5) is lower semicontinuous on $\mathrm{BV}(\Omega)$, with respect to the $L^{1}$-convergence.

We conclude this subsection, recalling a classical continuity result due to Reshetnyak (see [31] and, for instance, [3, Theorem 2.39]).

Theorem 2.3 Let $f: \Omega \times \mathbb{R}^{N} \rightarrow[0,+\infty)$ be a continuous function, positively 1 -homogeneus in the second variable and bounded in $\Omega \times \mathbb{S}^{N-1}$, where $\mathbb{S}^{N-1}$ is the unit sphere of $\mathbb{R}^{N}$. Then, for every sequence of measure $\left\{\mu_{h}\right\} \in \mathcal{M}\left(\Omega ; \mathbb{R}^{N}\right)$ strictly converging to a measure $\mu \in \mathcal{M}\left(\Omega ; \mathbb{R}^{N}\right)$, we have

$$
\lim _{h \rightarrow+\infty} \int_{\Omega} f\left(x, \frac{\mu_{h}}{\left|\mu_{h}\right|}(x)\right) d\left|\mu_{h}\right|(x)=\int_{\Omega} f\left(x, \frac{\mu}{|\mu|}(x)\right) d|\mu|(x),
$$

where $\frac{\mu_{h}}{\left|\mu_{h}\right|}$ is the Radon-Nikodym derivative of $\mu_{h}$ with respect to $\left|\mu_{h}\right|$ (respectively, $\frac{\mu}{|\mu|}$ is the Radon-Nikodým derivative of $\mu$ with respect to $\left.|\mu|\right)$.

\subsection{Relaxation and $\Gamma$-convergence}

For every $u \in \operatorname{BV}(\Omega)$, we define

$$
F(u)= \begin{cases}\int_{\Omega} f(x, u, \nabla u) d x & \text { if } u \in W^{1,1}(\Omega) \\ +\infty & \text { if } u \in \mathrm{BV}(\Omega) \backslash W^{1,1}(\Omega) .\end{cases}
$$

It is our aim to prove an integral representation theorem for the relaxation $\bar{F}$ of $F$, with respect to the $L^{1}$-convergence. We recall that the relaxation of $F$ is the greatest lower semicontinuous functional not greater than $F$; i.e., for every $u \in \mathrm{BV}(\Omega)$,

$$
\bar{F}(u):=\inf \left\{\liminf _{n \rightarrow+\infty} F\left(u_{n}\right): u_{n} \in W^{1,1}(\Omega), u_{n} \rightarrow u \text { in } L^{1}(\Omega)\right\} .
$$

For the sequel, we recall also that, given a sequence $\left\{F_{h}\right\}$ of functionals defined on $\operatorname{BV}(\Omega)$ of the type $(2.8)$, the functional $F^{\Gamma}:=\Gamma-\lim F_{h}$, with respect to the $L^{1}(\Omega)$-convergence, if it exists, is characterized by the following two inequalities: 
for every $u \in \operatorname{BV}(\Omega)$ and every $\left\{u_{h}\right\} \in W^{1,1}(\Omega)$, such that $u_{h} \rightarrow u$ strongly in $L^{1}(\Omega)$,

$$
F^{\Gamma}(u) \leq \liminf _{h \rightarrow+\infty} F_{h}\left(u_{h}\right)
$$

for every $u \in \operatorname{BV}(\Omega)$ there exists $\left\{\bar{u}_{h}\right\} \in W^{1,1}(\Omega)$, such that $\bar{u}_{h} \rightarrow u$ strongly in $L^{1}(\Omega)$ and

$$
F^{\Gamma}(u)=\lim _{h \rightarrow+\infty} F_{h}\left(\bar{u}_{h}\right)
$$

It is well known that $F^{\Gamma}$ is an $L^{1}$-lower semicontinuous functional on $\mathrm{BV}(\Omega)$ and, if $F_{h} \equiv F$ for every $h \in \mathbb{N}$, then the $\Gamma$-limit $F^{\Gamma}$ exists and it is equal to $\bar{F}$. Finally, a compactness result for the $\Gamma$-convergence holds; i.e., given a sequence $\left\{F_{h}\right\}$, up to a subsequence, there always exists the $\Gamma-\lim F_{h}$.

For other properties of the relaxation and $\Gamma$-convergence we refer to $[5,6,8,15,16]$.

\section{Main Results}

\subsection{A continuity theorem}

In this subsection we prove one of the main result of our paper, which concerns a continuity property for functionals defined on $\mathrm{BV}$, with respect to the strict convergence in $\mathrm{BV}$; i.e., the strong $L^{1}$-convergence of the functions and the convergence of the total variations of their derivatives. For the applications that we have in mind, we prefer to state this result in a space of arbitrary dimension $d>1$. Actually, in our case, $d=N+1$, as we will see in Proposition 3.9.

Let $\mathcal{O}$ be a bounded open subset of $\mathbb{R}^{d}$. Let $A: \mathcal{O} \times \mathbb{R} \rightarrow[0,+\infty)$ be a Borel function such that

$$
\begin{cases}(i) \quad A(\cdot, t) \in W^{1,1}(\mathcal{O}) \quad \text { and it is approximately continuous } \mathcal{H}^{d-1} \\ \text {-a.e. in } \mathcal{O}, \text { for every } t \in \mathbb{R}, \\ \text { for every bounded set } B \subset \mathbb{R} \text { there exists a constant } L(B) \text { such that } \\ \quad \int_{\mathcal{O}}\left|\nabla_{z} A\right|(z, t) d z<L(B) \quad \forall t \in B .\end{cases}
$$

Remark 3.1 Actually, the second requirement in (i) of (3.1) seems to be redundant, since it is well known that a $W^{1,1}$-function admits a representative, which is approximately continuous in $\mathcal{H}^{d-1}$-a.e. point of $\mathcal{O}$. Moreover, from the point of view of the functional, which we will consider in the following, it is not relevant the choice of the representative. However, we chose to identify $A(\cdot, t)$ with its precise representative, since, for our purpose, it is essential to assume that the hypotheses below hold exactly for this representative. 
We will assume

$$
\begin{aligned}
& \left|A(z, t)-A\left(z, t_{0}\right)\right| \leq \lambda\left|t-t_{0}\right| \quad \text { for every }(z, t),\left(z, t_{0}\right) \in \mathcal{O} \times \mathbb{R}, \\
& 0 \leq A(z, t) \leq \lambda \text { for every }(z, t) \in \mathcal{O} \times \mathbb{R},
\end{aligned}
$$

where $0<\lambda<+\infty$ is a fixed constant.

Remark 3.2 Actually, by using (3.2), it is easy to see that there exists $N_{0} \subset \mathcal{O}$ (independent of $t$ ), with $\mathcal{H}^{d-1}\left(N_{0}\right)=0$, such that $A(\cdot, t)$ is approximately continuous at $z_{0} \in \mathcal{O} \backslash N_{0}$, for every $t \in \mathbb{R}$; i.e.,

$$
\lim _{\varepsilon \rightarrow 0^{+}} \int_{Q\left(z_{0}, \varepsilon\right)}\left|A(z, t)-A\left(z_{0}, t\right)\right| d z=0 \quad \forall z_{0} \in \mathcal{O} \backslash N_{0} \quad \text { and } \quad \forall t \in \mathbb{R} .
$$

This implies that, for every $t \in \mathbb{R}, A(\cdot, t)$ coincides with its precise representative in $\mathcal{H}^{d-1}$-almost every $z \in \mathcal{O}$, independently of $t$.

Let $P: \mathcal{O} \times \mathbb{R}^{d} \rightarrow[0,+\infty)$ be a Borel function, such that

$$
\begin{gathered}
P(z, \cdot) \quad \text { is convex and positively } 1 \text {-homogeneous, for every } z \in \mathcal{O} \\
\quad 0 \leq P(z, \zeta) \leq \Lambda|\zeta| \text { for every }(z, \xi) \in \mathcal{O} \times \mathbb{R}^{d} \\
\left|P(z, \zeta)-P\left(z_{0}, \zeta\right)\right| \leq \Lambda|\zeta|\left|z-z_{0}\right| \text { for every }(z, \zeta),\left(z_{0}, \zeta\right) \in \mathcal{O} \times \mathbb{R}^{d}
\end{gathered}
$$

where $0<\Lambda<+\infty$ is a fixed constant. We note that, by (3.4) and (3.5), it easily follows that $P(z, \cdot)$ is Lipschitz continuous in $\zeta$, uniformly with respect to $z$. Hence, by virtue of $(3.6), P \in \mathcal{C}^{0}\left(\mathcal{O} \times \mathbb{R}^{d}\right)$.

Theorem 3.3 Assume that $A: \mathcal{O} \times \mathbb{R} \rightarrow[0,+\infty)$ and $P: \mathcal{O} \times \mathbb{R}^{d} \rightarrow[0,+\infty)$ are Borel functions satisfying (3.1)-(3.6). Let $\left\{v_{h}\right\}$ be a sequence in $\mathrm{BV}(\mathcal{O})$ such that there exists $v \in \mathrm{BV}(\mathcal{O})$ with $\left\{v_{h}\right\}$ strictly converging to $v$. Then

$$
\begin{gathered}
\lim _{h \rightarrow \infty} \int_{\mathcal{O}}\left[f_{v_{h}^{-}}^{v_{h}^{+}} A(z, t) d t\right] P\left(z, \frac{D v_{h}}{\left|D v_{h}\right|}(z)\right) d\left|D v_{h}\right|(z) \\
=\int_{\mathcal{O}}\left[f_{v^{-}}^{v^{+}} A(z, t) d t\right] P\left(z, \frac{D v}{|D v|}(z)\right) d|D v|(z) .
\end{gathered}
$$

Proof. Taking into account that $v_{h} \rightarrow v$ strongly in $L^{1}(\mathcal{O})$ and $\left|D v_{h}\right|(\mathcal{O}) \rightarrow$ $|D v|(\mathcal{O})$, it follows that $D v_{h} \rightarrow D v$ weakly* in $\mathcal{M}\left(\mathcal{O} ; \mathbb{R}^{d}\right)$ and so $\left\{D v_{h}\right\}$ strictly converges to $D v$. By Theorem 2.3 with $\Omega, \mathbb{R}^{N}$ and $f$ replaced by $\mathcal{O}, \mathbb{R}^{d}$ and $P$, respectively, and $\mu_{h}=D v_{h}, \mu=D v$, we have

$$
\lim _{h \rightarrow+\infty} \int_{\mathcal{O}} P\left(z, \frac{D v_{h}}{\left|D v_{h}\right|}(z)\right) d\left|D v_{h}\right|(z)=\int_{\mathcal{O}} P\left(z, \frac{D v}{|D v|}(z)\right) d|D v|(z) .
$$


Now we apply Theorem 2.2 with $f(z, t, \zeta)=A(z, t) P(z, \zeta)$ and $f(z, t, \zeta)$ $=[\lambda-A(z, t)] P(z, \zeta)$, respectively. In fact, taking into account the positive 1-homogeneity of $P(z, \cdot)$, we have $f^{\infty}(z, t, \zeta)=f(z, t, \zeta)$; moreover, by our assumptions and Remark 3.2, we have that hypotheses (i)-(iii) of Theorem 2.2 hold. Therefore, we obtain

$$
\begin{array}{r}
\liminf _{h \rightarrow+\infty} \int_{\mathcal{O}}\left[\int_{v_{h}^{-}}^{v_{h}^{+}} A(z, t) d t\right] P\left(z, \frac{D v_{h}}{\left|D v_{h}\right|}(z)\right) d\left|D v_{h}\right|(z) \\
\geq \int_{\mathcal{O}}\left[f_{v^{-}}^{v^{+}} A(z, t) d t\right] P\left(z, \frac{D v}{|D v|}(z)\right) d|D v|(z)
\end{array}
$$

and

$$
\begin{aligned}
\liminf _{h \rightarrow+\infty} \int_{\mathcal{O}} & {\left[f_{v_{h}^{-}}^{v_{h}^{+}}(\lambda-A(z, t)) d t\right] P\left(z, \frac{D v_{h}}{\left|D v_{h}\right|}(z)\right) d\left|D v_{h}\right|(z) } \\
& \geq \int_{\mathcal{O}}\left[f_{v^{-}}^{v^{+}}(\lambda-A(z, t)) d t\right] P\left(z, \frac{D v}{|D v|}(z)\right) d|D v|(z)
\end{aligned}
$$

where, due to the positive 1-homogeneity of $P$, the functionals are written in the abbreviated form as in 2.6.

Combining (3.8), (3.9) and (3.10), the thesis follows.

Remark 3.4 We point out that the previous theorem is obtained by using the result of lower semicontinuity in [11] whose proof is heavily based on the convexity of the integrand with respect to the last variable. Since we need to apply that result, it is crucial to impose the separation of the variables, in order to assure the convexity also of the integrand appearing in (3.10).

We note that it cannot be expected that the previous result could hold for a strictly converging sequence of general measures, as it happens for the Reshetnyak Continuity Theorem, where the integrand continuously depends on $x$. The following example remarks this fact.

Example 3.5 Let $\Omega \subseteq \mathbb{R}^{N}$ be a bounded set. Let $\left\{x_{h}\right\} \subseteq \Omega$ be a sequence converging to $x_{0} \in \Omega$. For every $h \in \mathbb{N}$, let $\mu_{h}$ and $\mu_{0}$ be the Dirac mass concentrated in $x_{h}$ and in $x_{0}$, respectively. Clearly, $\left\{\mu_{h}\right\}$ strictly converges to $\mu_{0}$; moreover, if $a: \Omega \rightarrow[0,+\infty)$ is a Borel function and

$$
F(\mu)=\int_{\Omega} a(x) d|\mu| \quad \forall \mu \in \mathcal{M}(\Omega),
$$

it is easy to see that $F\left(\mu_{h}\right) \rightarrow F\left(\mu_{0}\right)$ if and only if $a\left(x_{h}\right) \rightarrow a\left(x_{0}\right)$. Since this must hold for every $x_{0} \in \Omega$ and every $x_{h} \rightarrow x_{0}, a$ must be continuous. 


\subsection{The relaxation theorem}

Let $a: \Omega \times \mathbb{R} \rightarrow[0,+\infty)$ be a Borel function such that

$$
\left\{\begin{array}{l}
(\text { i }) \quad a(\cdot, s) \in W^{1,1}(\Omega) \text { and it is approximately continuous } \mathcal{H}^{N-1} \\
\text {-a.e. in } \Omega, \text { for every } s \in \mathbb{R}, \\
\text { for every bounded set } B \subset \mathbb{R} \text { there exists a constant } L(B) \text { such that } \\
\int_{\Omega}\left|\nabla_{x} a\right|(x, s) d x<L(B) \quad \forall s \in B .
\end{array}\right.
$$

Remark 3.6 As in Remark 3.1, we point out that the second requirement in (i) of (3.11) is essential to identify $a(\cdot, s)$ with its precise representative, so that the hypotheses below hold exactly for this representative.

We will assume that

$$
\begin{gathered}
\left|a(x, s)-a\left(x, s_{0}\right)\right| \leq \lambda\left|s-s_{0}\right| \text { for every }(x, s),\left(x, s_{0}\right) \in \Omega \times \mathbb{R}, \\
0 \leq a(x, s) \leq \lambda \text { for every }(x, s) \in \Omega \times \mathbb{R},
\end{gathered}
$$

where $0<\lambda<+\infty$ is a fixed constant.

Remark 3.7 As in Remark 3.2, by using (3.12), it is easy to see that there exists $N_{0} \subset \Omega$ (independent of $s$ ), with $\mathcal{H}^{N-1}\left(N_{0}\right)=0$, such that $a(\cdot, s)$ is approximately continuous at $x_{0} \in \Omega \backslash N_{0}$, for every $s \in \mathbb{R}$.

Let $p: \mathbb{R} \times \mathbb{R}^{N} \rightarrow[0,+\infty)$ be a Borel function satisfying the following properties:

$$
\begin{aligned}
& p(s, \cdot) \quad \text { is convex, for every } s \in \mathbb{R}, \\
& \left|p(s, \xi)-p\left(s_{0}, \xi\right)\right| \leq \Lambda(1+|\xi|)\left|s-s_{0}\right| \\
& \quad \text { for every }(s, \xi),\left(s_{0}, \xi\right) \in \mathbb{R} \times \mathbb{R}^{N}, \\
& 0 \leq p(s, \xi) \leq \Lambda(1+|\xi|) \quad \text { for every }(s, \xi) \in \mathbb{R} \times \mathbb{R}^{N},
\end{aligned}
$$

where $0<\Lambda<+\infty$ is a fixed constant. By (3.14) and (3.16), it follows that $p(s, \cdot)$ is Lipschitz continuous in $\xi$, uniformly with respect to $s$.

We consider, for every $u \in \operatorname{BV}(\Omega)$, the functional defined in (2.5), with $f(x, s, \xi)=$ $a(x, s) p(s, \xi)$ and $f^{\infty}(x, s, \xi)=a(x, s) p^{\infty}(s, \xi)$, where, by (i) of (3.11) and Remark $3.7, a(\cdot, s)$ coincides with its precise representative, independently of $s \in \mathbb{R}$, and $p^{\infty}: \mathbb{R} \times \mathbb{R}^{N} \rightarrow[0,+\infty)$ is the recession function of $p$ (see (2.2)). We recall that $p^{\infty}$ is a Borel function, which is convex and positively 1-homogeneus with respect to $\xi$; moreover,

$$
\begin{gathered}
\left|p^{\infty}(s, \xi)-p^{\infty}\left(s_{0}, \xi\right)\right| \leq \Lambda|\xi|\left|s-s_{0}\right| \quad \text { for every }(s, \xi),\left(s_{0}, \xi\right) \in \mathbb{R} \times \mathbb{R}^{N}, \\
0 \leq p^{\infty}(s, \xi) \leq \Lambda|\xi| \text { for every }(s, \xi) \in \mathbb{R} \times \mathbb{R}^{N} .
\end{gathered}
$$


We will prove that

$$
\bar{F}(u)=\mathcal{F}(u) \quad \forall u \in \operatorname{BV}(\Omega),
$$

where $F$ is defined in $(2.8)$, with $f(x, s, \xi)=a(x, s) p(s, \xi)$.

As pointed out in the introduction, under assumptions of more regularity of the integrand function $a$, formula (3.19) was already proven, for istance, in [9] and [19], hence it defines a "natural" extension of $F(u)=\int_{\Omega} a(x, u) p(u, \nabla u) d x$ from $W^{1,1}(\Omega)$ to $\mathrm{BV}(\Omega)$. In the next theorem we state that this result still holds under our weaker assumptions, up to assume the separation of the variables.

Theorem 3.8 Assume that $a: \Omega \times \mathbb{R} \rightarrow[0,+\infty)$ and $p: \mathbb{R} \times \mathbb{R}^{N} \rightarrow[0,+\infty)$ are Borel functions satisfying (3.11)-(3.16). Let $F: \operatorname{BV}(\Omega) \rightarrow[0,+\infty]$ be the functional defined in (2.8), with $f(x, s, \xi)=a(x, s) p(\xi)$, and $\bar{F}$ be the relaxation of F. Then formula (3.19) holds; i.e.,

$$
\begin{aligned}
\bar{F}(u)= & \int_{\Omega} a(x, u) p(u, \nabla u) d x+\int_{\Omega} a(x, \widetilde{u}) p^{\infty}\left(\widetilde{u}, D^{c} u\right) \\
& +\int_{S_{u} \cap \Omega}\left(\int_{u^{-}(x)}^{u^{+}(x)} a(x, s) p^{\infty}\left(s, \nu_{u}\right) d s\right) d \mathcal{H}^{N-1}(x)
\end{aligned}
$$

for every $u \in \mathrm{BV}(\Omega)$.

A crucial tool in order to prove this theorem is the continuity Theorem 3.3, which is adapted to our particular case in the following proposition.

Proposition 3.9 Let $\Omega \subset \mathbb{R}^{N}$ a bounded open set, $M>0$ and $\mathcal{O}=\Omega \times$ $(-M, M) \subseteq \mathbb{R}^{N+1}$. Assume that $\bar{a}: \Omega \times \mathbb{R} \rightarrow[0,+\infty)$ is a Borel function which is equal to zero out of $\mathcal{O}$ and satisfies (3.11), (3.12) with $\Omega \times \mathbb{R}$ replaced by $\mathcal{O}$, and (3.13). Let $\bar{p}: \mathbb{R} \times \mathbb{R}^{N+1} \rightarrow[0,+\infty)$ be a Borel function, which is equal to zero out of $(-M, M) \times \mathbb{R}^{N+1}$, convex and positively 1-homogeneous in $\xi$, satisfying

$$
\begin{aligned}
\left|\bar{p}(s, \zeta)-\bar{p}\left(s_{0}, \zeta\right)\right| & \leq \Lambda|\zeta|\left|s-s_{0}\right| \quad \text { for every } \\
(s, \zeta),\left(s_{0}, \zeta\right) & \in(-M, M) \times \mathbb{R}^{N+1} \\
0 & \leq \bar{p}(s, \zeta) \leq \Lambda|\zeta| \quad \text { for every } \zeta \in \mathbb{R}^{N+1}
\end{aligned}
$$

for a proper real constant $\Lambda>0$. Let $\left\{v_{h}\right\}$ be a sequence in $\mathrm{BV}(\mathcal{O})$ such that there exists $v \in \mathrm{BV}(\mathcal{O})$ with $\left\{v_{h}\right\}$ strictly converging to $v$. Then

$$
\begin{gathered}
\lim _{h \rightarrow \infty} \int_{\mathcal{O}} \bar{a}(x, s) \bar{p}\left(s, \frac{D v_{h}}{\left|D v_{h}\right|}(x, s)\right) d\left|D v_{h}\right|(x, s) \\
=\int_{\mathcal{O}} \bar{a}(x, s) \bar{p}\left(s, \frac{D v}{|D v|}(x, s)\right) d|D v|(x, s) .
\end{gathered}
$$


Proof. We will apply Theorem 3.3, with $z=(x, s), A(z, t)=\bar{a}(x, s)$ and $P(z, \zeta)$ $=\bar{p}(s, \zeta)$. Firstly we prove (3.1). In order to prove that $\bar{a} \in W^{1,1}(\mathcal{O})$, it is sufficient to remark that, by $(3.13), \bar{a} \in L^{\infty}(\mathcal{O})$ and, by $(3.12), \bar{a}(x, \cdot)$ is a Lipschitz continuous function. Then, we have

$$
\begin{aligned}
\int_{\mathcal{O}}\left|\nabla_{(x, s)} \bar{a}\right|(x, s) d x d s & \leq \int_{(-M, M)}\left[\int_{\Omega}\left|\nabla_{x} \bar{a}\right|(x, s) d x+\int_{\Omega}\left|\frac{\partial \bar{a}}{\partial s}(x, s)\right| d x\right] d s \\
& \leq 2 M(L+|\Omega| \lambda)
\end{aligned}
$$

where we used (3.11), with $L=L(B)$ and $B=(-M, M)$. In order to prove that $\bar{a}$ is approximately continuous $\mathcal{H}^{N}$-a.e. in $\mathcal{O}$, set $N=N_{0} \times(-M, M)$, where $N_{0}$ is defined in Remark 3.7, and note that $\mathcal{H}^{N}(N)=0$. Moreover, for every $\left(x_{0}, s_{0}\right) \in \mathcal{O} \backslash N$, we have

$$
\begin{aligned}
& \lim _{\varepsilon \rightarrow 0^{+}} f_{s_{0}-\varepsilon}^{s_{0}+\varepsilon} f_{B_{\varepsilon}\left(x_{0}\right)}\left|\bar{a}(x, s)-\bar{a}\left(x_{0}, s_{0}\right)\right| d x d s \\
& \quad \leq \lambda \lim _{\varepsilon \rightarrow 0^{+}} f_{s_{0}-\varepsilon}^{s_{0}+\varepsilon}\left|s-s_{0}\right| d s+\lim _{\varepsilon \rightarrow 0^{+}} f_{B_{\varepsilon}\left(x_{0}\right)}\left|\bar{a}\left(x, s_{0}\right)-\bar{a}\left(x_{0}, s_{0}\right)\right| d x=0 .
\end{aligned}
$$

Finally, since $\bar{a}$ does not depend on $t$ and $\bar{p}$ depends only $s$, it is easy to verify that all the other hypotheses of Theorem 3.3 are satisfied. Hence, by (3.7) the thesis follows.

By previous proposition, we obtain the following result.

Theorem 3.10 Assume that $a: \Omega \times \mathbb{R} \rightarrow[0,+\infty)$ and $p: \mathbb{R} \times \mathbb{R}^{N} \rightarrow[0,+\infty)$ are Borel function satisfying (3.11)-(3.16). Then, for every $u \in \mathrm{BV}(\Omega)$, we have $\mathcal{F}(u) \geq \bar{F}(u)$.

Proof. Let $u \in \operatorname{BV}(\Omega) \cap L^{\infty}(\Omega)$ and $u_{h}=u * \phi_{h}$, where $\left\{\phi_{h}\right\}$ is a sequence of mollifiers. Then $\left\{u_{h}\right\} \subseteq W^{1,1}(\Omega) \cap L^{\infty}(\Omega)$ and $\left\|u_{h}\right\|_{\infty}<M$, where $M:=\|u\|_{\infty}+1$. Moreover, $u_{h} \rightarrow u$ strongly in $L^{1}$ and $\int_{\Omega}\left|\nabla u_{h}\right| d x \rightarrow|D u|(\Omega)$. By Theorems 1.8 and 1.10 in [30] and Proposition 1.1 in [7], the sequence $\left\{\chi_{\mathcal{G}\left(u_{h}\right)}\right\}$ strictly converges to $\chi_{\mathcal{G}(u)}$ in $\mathcal{O}=\Omega \times(-M, M)$; i.e.,

$$
\chi_{\mathcal{G}\left(u_{h}\right)} \rightarrow \chi_{\mathcal{G}(u)} \quad \text { strongly in } L^{1}(\mathcal{O}) \quad \text { and } \quad\left|\alpha\left(u_{h}\right)\right|(\mathcal{O}) \rightarrow|\alpha(u)|(\mathcal{O}),
$$

where $\alpha\left(u_{h}\right)=D \chi_{\mathcal{G}\left(u_{h}\right)}$ and $\alpha(u)=D \chi_{\mathcal{G}(u)}$. Set

$$
a_{M}(x, s)=\left\{\begin{array}{lll}
a(x, s) & \text { if } \quad|s|<M \\
0 & \text { if } \quad|s| \geq M,
\end{array} \quad p_{M}(s, \xi)=\left\{\begin{array}{lll}
p(s, \xi) & \text { if } & |s|<M \\
0 & \text { if } & |s| \geq M
\end{array}\right.\right.
$$


By Lemma 2.1, with $f(x, s, \xi)=a_{M}(x, s) p_{M}(s, \xi)$, we have that, for every $h \in \mathbb{N}$

$$
\begin{aligned}
F\left(u_{h}\right) & =\mathcal{F}\left(u_{h}\right)=\int_{\Omega} a_{M}\left(x, u_{h}\right) p_{M}\left(u_{h}, \nabla u_{h}\right) d x \\
& =\int_{\Omega \times \mathbb{R}} a_{M}(x, s) \widehat{p_{M}}\left(s, \frac{\alpha\left(u_{h}\right)}{\left|\alpha\left(u_{h}\right)\right|}(x, s)\right) d\left|\alpha\left(u_{h}\right)\right|(x, s) \\
& =\int_{\mathcal{O}} a_{M}(x, s) \widehat{p_{M}}\left(s, \frac{\alpha\left(u_{h}\right)}{\left|\alpha\left(u_{h}\right)\right|}(x, s)\right) d\left|\alpha\left(u_{h}\right)\right|(x, s),
\end{aligned}
$$

where $\widehat{p_{M}}$ is the function convex and positively 1-homogeneus in $(\xi, \tau)$ defined in (2.4), and in the second equality of (3.23) we take into account that $\left\|u_{h}\right\|_{\infty}<M$. Let us note that, by (3.15) and (3.16), it follows

$$
\begin{gathered}
\left|\widehat{p_{M}}(s, \xi, \tau)-\widehat{p_{M}}\left(s_{0}, \xi, \tau\right)\right| \leq \widehat{\Lambda}(|\xi|+|\tau|)\left|s-s_{0}\right| \\
\forall(s, \xi, \tau),\left(s_{0}, \xi, \tau\right) \in(-M, M) \times \mathbb{R}^{N} \times(-\infty, 0], \\
0 \leq \widehat{p_{M}}(s, \xi, \tau) \leq \widehat{\Lambda}|(\xi, \tau)| \quad \forall(s, \xi, \tau) \in(-M, M) \times \mathbb{R}^{N} \times(-\infty, 0],
\end{gathered}
$$

with a suitable constant $\widehat{\Lambda}>0$. By (3.23) and Proposition 3.9 with $\bar{a}=a_{M}$, $\bar{p}=\widehat{p_{M}}$ and $v_{h}=\chi_{\mathcal{G}\left(u_{h}\right)}$ (so that $\left.D v_{h}=\alpha\left(u_{h}\right)\right)$, we obtain

$$
\begin{aligned}
\lim _{h \rightarrow \infty} F\left(u_{h}\right) & =\lim _{h \rightarrow \infty} \mathcal{F}\left(u_{h}\right) \\
& =\lim _{h \rightarrow \infty} \int_{\mathcal{O}} a_{M}(x, s) \widehat{p_{M}}\left(s, \frac{\alpha\left(u_{h}\right)}{\left|\alpha\left(u_{h}\right)\right|}(x, s)\right) d\left|\alpha\left(u_{h}\right)\right|(x, s) \\
& =\int_{\mathcal{O}} a_{M}(x, s) \widehat{p_{M}}\left(s, \frac{\alpha(u)}{|\alpha(u)|}(x, s)\right) d|\alpha(u)|(x, s)=\mathcal{F}(u) .
\end{aligned}
$$

Then, by the lower semicontinuity of the relaxed functional, it follows

$$
\bar{F}(u) \leq \liminf _{h \rightarrow \infty} \bar{F}\left(u_{h}\right) \leq \lim _{h \rightarrow \infty} F\left(u_{h}\right)=\mathcal{F}(u)
$$

for every $u \in \mathrm{BV}(\Omega) \cap L^{\infty}(\Omega)$. To conclude the proof, we take $u \in \operatorname{BV}(\Omega)$ and proceed by a standard truncation argument. For every $k>0$, define $u_{k}=(u \wedge k) \vee(-k)$. Then, $u_{k} \in \mathrm{BV}(\Omega) \cap L^{\infty}(\Omega),\left\|u_{k}\right\|_{\infty} \leq k$ and (see, for instance, [3, Theorem 3.99])

while

$$
\nabla u_{k} d x+D^{c} u_{k}= \begin{cases}\nabla u d x+D^{c} u & \text { if }|u|<k \\ 0 & \text { if }|u| \geq k\end{cases}
$$

$$
\left(u_{k}^{+}-u_{k}^{-}\right) \nu_{u_{k}} d \mathcal{H}^{N-1}\left\lfloor J_{u_{k}}=\left(u_{k}^{+}-u_{k}^{-}\right) \nu_{u} d \mathcal{H}^{N-1}\left\lfloor J_{u},\right.\right.
$$


and $u_{k}^{+}-u_{k}^{-} \leq u^{+}-u^{-}$. Moreover, $u_{k} \rightarrow u$ strongly in $L^{1}(\Omega)$. By (3.25) we obtain

$$
\begin{aligned}
\bar{F}(u) \leq & \liminf _{k \rightarrow \infty} \bar{F}\left(u_{k}\right) \leq \liminf _{k \rightarrow \infty} \mathcal{F}\left(u_{k}\right) \\
\leq & \liminf _{k \rightarrow \infty}\left\{\int_{\Omega \cap\{|u| \leq k\}} a(x, u) p(u, \nabla u) d x+C \mathcal{L}^{N}(\Omega \cap\{|u|>k\})\right. \\
& +\int_{\Omega \cap\{|u| \leq k\}} a(x, \widetilde{u}) p^{\infty}\left(\widetilde{u}, D^{c} u\right) \\
& \left.+\int_{\Omega}\left(\int_{u_{k}^{-}}^{u_{k}^{+}} a(x, s) p^{\infty}\left(s, \nu_{u}\right) d s\right) d \mathcal{H}^{N-1}(x)\right\} \leq \mathcal{F}(u) .
\end{aligned}
$$

Hence the thesis is achieved.

Proof of Theorem 3.8 Let us note that all the assumptions of Theorem 2.2 are satisfied for $f(x, s, \xi)=a(x, s) p(s, \xi)$. Hence, the functional $\mathcal{F}$, defined in $(2.5)$ is $L^{1}$-lower semicontinuous on $\mathrm{BV}(\Omega)$ and $\mathcal{F}(u) \leq F(u)$ for every $u \in \mathrm{BV}(\Omega)$; therefore, by using the properties of the relaxation, we have that

$$
\mathcal{F}(u) \leq \bar{F}(u)
$$

for every $u \in \mathrm{BV}(\Omega)$. The opposite inequality follows by Theorem 3.10 .

\section{$3.3 \quad \Gamma$-convergence and pointwise convergence.}

In this subsection we state a $\Gamma$-convergence result for a sequence of integral functionals, whose integrands pointwise converge to an integrand, which is not necessarily continuous with respect to $x$ nor coercive. Similar results in the continuous and coercive case can be found in [9] (see also [8]).

Theorem 3.11 Let $a_{h}: \Omega \times \mathbb{R} \rightarrow[0,+\infty)$ be a sequence of Borel functions such that

$$
0 \leq a_{h}(x, s) \leq \lambda \quad \text { for every }(x, s) \in \Omega \times \mathbb{R},
$$

where $0<\lambda<+\infty$ is a fixed constant. Let $p_{h}: \mathbb{R} \times \mathbb{R}^{N} \rightarrow[0,+\infty)$ be a sequence Borel functions satisfying the following properties:

$$
0 \leq p_{h}(s, \xi) \leq \Lambda(1+|\xi|) \quad \text { for every }(s, \xi) \in \mathbb{R} \times \mathbb{R}^{N},
$$

where $0<\Lambda<+\infty$ is a fixed constant. For every $u \in \mathrm{BV}(\Omega)$, we define

$$
F_{h}(u)= \begin{cases}\int_{\Omega} a_{h}(x, u) p_{h}(u, \nabla u) d x & \text { if } u \in W^{1,1}(\Omega) \\ +\infty & \text { if } u \in \operatorname{BV}(\Omega) \backslash W^{1,1}(\Omega) .\end{cases}
$$


Assume that $\left\{a_{h}\right\}$ converges pointwise to a Borel function a $: \Omega \times \mathbb{R} \rightarrow[0,+\infty)$, satisfying (3.11)-(3.13), and that $\left\{p_{h}\right\}$ converges pointwise to a Borel function $p$ : $\mathbb{R} \times \mathbb{R}^{N} \rightarrow[0,+\infty)$ satisfying $(3.14)-(3.16)$. Finally, let $\left\{\varepsilon_{h}\right\}$ be an infinitesimal sequence, such that

$\left(1+\varepsilon_{h}\right) a_{h}(x, s) p_{h}(s, \xi) \geq a(x, s) p(s, \xi)-\varepsilon_{h} \quad \forall(x, s, \xi) \in \Omega \times \mathbb{R} \times \mathbb{R}^{N}, \forall h \in \mathbb{N}$.

Then, for every $u \in \mathrm{BV}(\Omega)$, we have

$$
\left(\Gamma_{h \rightarrow+\infty}-\lim _{h} F_{h}\right)(u)=\mathcal{F}(u) .
$$

Proof. Up to take a subsequence, still denoted by $\left\{F_{h}\right\}$, we may assume that there exists the $\Gamma$-limit of $\left\{F_{h}\right\}$. Given $u \in W^{1,1}(\Omega)$, by (3.29) we obtain that, for every $h \in \mathbb{N}, F_{h}(u) \geq F(u)-\varepsilon_{h}\left[|\Omega|+F_{h}(u)\right]$, where $F$ is defined in (2.8), with $f(x, s, \xi)=a(x, s) p(s, \xi)$. Hence, by (2.10) we have that, for every $u \in \operatorname{BV}(\Omega)$, there exists $\left\{\bar{u}_{h}\right\} \subset W^{1,1}(\Omega)$ such that $\bar{u}_{h} \rightarrow u$ strongly in $L^{1}(\Omega)$ and

$$
\left(\Gamma-\lim _{h \rightarrow+\infty} F_{h}\right)(u)=\lim _{h \rightarrow+\infty} F_{h}\left(\bar{u}_{h}\right) .
$$

Clearly, we may assume that the previous limit is finite (otherwise the conclusion below is trivial). Taking into account also Theorem 3.8, it follows

$$
\begin{aligned}
\left(\Gamma_{h \rightarrow+\infty} \lim _{h}\right)(u) & =\lim _{h \rightarrow+\infty} F_{h}\left(\bar{u}_{h}\right) \\
& \geq \liminf _{h \rightarrow+\infty} F\left(\bar{u}_{h}\right)-\lim _{h \rightarrow+\infty} \varepsilon_{h}\left[|\Omega|+F_{h}\left(\bar{u}_{h}\right)\right] \geq \bar{F}(u)=\mathcal{F}(u)
\end{aligned}
$$

In order to prove the opposite inequality, we note that, by the Dominated Convergence Theorem, property (2.9) and taking into account the lower semicontinuity of the $\Gamma$-limit, it follows

$$
\left(\Gamma_{h \rightarrow+\infty} \lim _{h}\right)(u) \leq \bar{F}(u)=\mathcal{F}(u) \quad \forall u \in \mathrm{BV}(\Omega),
$$

where the last equality is due to Theorem 3.8. Then, the whole sequence $\left\{F_{h}\right\}$, and not only a subsequence, $\Gamma$-converges and the thesis is achieved.

\subsection{A counterexample}

In this subsection we present an example which shows that, in order to obtain the Continuity Theorem 3.3, it is necessary some control of the gradient of the integrand with respect to $x$. In fact, the functional we will construct below has an integrand which satisfies all the assumptions of Theorem 3.3, apart from the $W^{1,1}$-dependence with respect to $x$, and for it the formula (3.7) does not hold. 
Let $\Omega=(-1,1)$ and for every $k=3,4, \ldots$ let $E_{k}=\left(\frac{1}{2^{k+1}}, \frac{1}{2^{k}}-\frac{1}{4^{k}}\right)$ and $E_{k}^{\prime}=\left(\frac{1}{2^{k+1}}+\frac{1}{4^{k}}, \frac{1}{2^{k}}-\frac{2}{4^{k}}\right)$. We consider a continuous piecewise linear function $\beta_{k}(x)$ such that $0 \leq \beta_{k}(x) \leq 1, \beta_{k}(x)$ is equal to 1 in $E_{k}^{\prime}$ and it is equal to 0 in $(-1,1) \backslash E_{k}$. Set $\beta(x)=\chi_{(-1,0]}(x)+\sum_{k=3}^{\infty} \beta_{k}(x)$. We remark that by definition, for every $x \neq 0$ the function $\beta$ is continuous in $x$. Moreover $\beta$ is approximately continuous in 0 ; i.e.

$$
\lim _{\rho \rightarrow 0^{+}} \frac{1}{2 \rho} \int_{-\rho}^{\rho}|\beta(x)-\beta(0)| d x=0 .
$$

In fact, by taking for simplicity $\rho=\frac{1}{2^{n}}$,

$$
\begin{aligned}
\lim _{n \rightarrow+\infty} \frac{2^{n}}{2} \int_{-\frac{1}{2^{n}}}^{\frac{1}{2^{n}}}|\beta(x)-1| d x & =\lim _{n \rightarrow+\infty} \frac{2^{n}}{2} \int_{0}^{\frac{1}{2^{n}}}|\beta(x)-1| d x \\
& =\lim _{n \rightarrow+\infty} \frac{2^{n}}{2} \sum_{k=n}^{+\infty} \int_{\frac{1}{2^{k+1}}}^{\frac{1}{2^{k}}}\left(1-\beta_{k}(x)\right) d x \\
& \leq \lim _{n \rightarrow+\infty} \frac{2^{n}}{2} \sum_{k=n}^{+\infty}\left[\int_{\frac{1}{2^{k+1}}}^{\frac{1}{2^{k+1}}+\frac{1}{4^{k}}} d x+\int_{\frac{1}{2^{k}}-\frac{2}{4^{k}}}^{\frac{1}{2^{k}}} d x\right] \\
& =\lim _{n \rightarrow \infty} \frac{2^{n}}{2} \sum_{k=n}^{+\infty}\left(\frac{1}{4^{k}}+\frac{2}{4^{k}}\right)=0 .
\end{aligned}
$$

Finally we note that $\beta \in L^{1}(\Omega)$, but $\beta \notin W^{1,1}(\Omega)$ (actually $\beta \notin \mathrm{BV}(\Omega)$ ). Now, let us define the functional

$$
\mathcal{F}(u)=\int_{\Omega} \beta(x) d|D u|(x) \quad \forall u \in \operatorname{BV}(\Omega),
$$

which coincides with the functional in (3.7) with $z=(x, s), \zeta=\xi, A(z, t)$ $=\beta(x)$ and $P(z, \zeta)=|\xi|$. We remark that for this functional all the hypotheses of Theorem 3.3 are satisfied, except of the control in the gradient of $\beta$, but we will prove that there exists a sequence $u_{k} \in \mathrm{BV}(\Omega)$ strictly converging to a function $u \in \mathrm{BV}(\Omega)$ such that

$$
\mathcal{F}(u) \neq \lim _{k \rightarrow+\infty} \mathcal{F}\left(u_{k}\right)
$$

Indeed, for every $k \geq 3$, let us consider the function $u_{k} \in \mathrm{BV}(\Omega)$ defined by

$$
u_{k}(x)= \begin{cases}1 & \text { if } x \in\left(-1, \frac{1}{2^{k}}-\frac{1}{2^{2 k+1}}\right), \\ 0 & \text { if } x \in\left(\frac{1}{2^{k}}-\frac{1}{2^{2 k+1}}, 1\right) .\end{cases}
$$

It is easy to check that $u_{k} \rightarrow u:=\chi_{(-1,0)}$ strictly in $\operatorname{BV}(\Omega)$. Nevertheless,

$$
\lim _{k \rightarrow+\infty} \mathcal{F}\left(u_{k}\right)=\lim _{k \rightarrow+\infty} \beta\left(\frac{1}{2^{k}}-\frac{1}{2^{2 k+1}}\right)=0,
$$

while $\mathcal{F}(u)=\beta(0)=1$. 


\section{References}

[1] M. AMAR and G. BELLETTINI, A notion of total variation depending on a metric with discontinuous coefficients, Annales de l'Institute Henri Poincaré, Analyse Non Linéaire 11(1) (1994), 91-133.

[2] M. AMAR, V. DE CICCO and N. FUSCO, A relaxation result in BV for integral functionals with discontinuous integrands. ESAIM: Cocv 13 (2007), $396-412$.

[3] L. AMBROSIO, N. FUSCO and D. PALLARA, Functions of bounded variation and free discontinuity problems, Oxford University Press, New York, (2000).

[4] G. BOUCHITTÉ and G. DAL MASO, Integral representation and relaxation of convex local functionals on BV $(\Omega)$, Ann. Sc. Norm. Super. Pisa, Cl. Sci. IV Serie 20(4) (1993), 483-533.

[5] A. BRAIDES, Г-convergence for beginners. Oxford University Press, New York, (2002).

[6] G. BUTTAZZO, Semicontinuity, Relaxation and Integral Representation Problems in the Calculus of Variations. Pitman Res. Notes in Math., Longman, Harlow, (1989).

[7] M. CARRIERO, G. DAL MASO, A. LEACI and E. PASCALI, Relaxation of the non-parametric Plateau problem with an obstacle, J. Math. Pures et Appl. 67 (1988), 359-396.

[8] G. DAL MASO, An Introduction to $\Gamma$-convergence. Birkhäuser, Boston, (1993).

[9] G. DAL MASO, Integral representation on $\mathrm{BV}(\Omega)$ of $\Gamma$-limits of variational integrals, Manuscripta Math. 30 (1980), 387-416.

[10] V. DE CICCO, Lower semicontinuity for certain integral functionals on BV $(\Omega)$, Boll. U.M.I. 5-B (1991), 291-313.

[11] V. DE CICCO, N. FUSCO and A. VERDE, On $L^{1}$-lower semicontinuity in $\mathrm{BV}(\Omega)$, J. of Convex Analysis 12 (2005), 173-185.

[12] V. DE CICCO, N. FUSCO and A. VERDE, A chain rule formula in $\operatorname{BV}(\Omega)$ and its applications to lower semicontinuity. CALC. VAR. PARTIAL DIFFER. EQU. 28(41) (2007), 427-447.

[13] V. DE CICCO and G. LEONI, A chain rule in $L^{1}(\operatorname{div} ; \Omega)$ and its applications to lower semicontinuity, Calc. Var. Partial Differ. Equ. 19 (2004), 23-51. 
[14] E. DE GIORGI, G. BUTTAZZO and G. DAL MASO, On the lower semicontinuity of certain integral functionals, Atti Accad. Naz. Lincei Rend. Cl. Sci. Fis. Mat. Natur. 74 (1983), 274-282.

[15] E. DE GIORGI and T. FRANZONI, Su un tipo di convergenza variazionale, Atti Accad. Naz. Lincei Rend. Cl. Sci. Fis. Mat. Natur. 58 (1975), 842-850.

[16] E. DE GIORGI and T. FRANZONI, Su un tipo di convergenza variazionale, Rend. Sem. Mat. Brescia 3 (1979), 63-101.

[17] L. C. EVANS and R. F. GARIEPY, Lecture Notes on Measure Theory and Fine Properties of Functions. CRC Press, Boca Raton, (1992).

[18] H. FEDERER, Geometric Measure Theory. Springer-Verlag, Berlin, (1969).

[19] I. FONSECA and G. LEONI, Some remarks on lower semicontinuity, Indiana Univ. Math. J. 49 (2000), 617-635.

[20] I. FONSECA and G. LEONI, On lower semicontinuity and relaxation, Proc. R. Soc. Edinb., Sect. A, Math. 131 (2001), 519-565.

[21] N. FUSCO, F. GIANNETTI and A. VERDE, A remark on the $L^{1}$-lower semicontinuity for integral functionals in BV, Manuscripta Math. 112 (2003), $313-323$.

[22] N. FUSCO, M. GORI and F. MAGGI, A remark on Serrin's Theorem. (To appear).

[23] M. GIAQUINTA, G. MODICA and J. SOUC̆EK, Cartesian Currents in the Calculus of Variations, Part I: Cartesian Current, Part II: Variational Integrals. Springer, (1998).

[24] E. GIUSTI, Minimal Surfaces and Functions of Bounded Variation. Birkhäuser, Boston, (1984).

[25] M. GORI and P. MARCELLINI, An extension of the Serrin's lower semicontinuity theorem, J. of Convex Anal. 9(2) (2002), 475-502.

[26] M. GORI and F. MAGGI, The common root of the geometric conditions in Serrin's lower semicontinuity theorem, Ann. Mat. Pura Appl. 184(1) (2005) 95-114.

[27] M. GORI, F. MAGGI and P. MARCELLINI, On some sharp conditions for lower semicontinuity in $L^{1}$, Diff. Int. Eq. 16 (2003), 51-76.

[28] F. MAGGI, On the relaxation on BV of certain non coercive integral functionals, J. Convex Anal. 10 (2003), 477-489.

[29] V. G. MAZ'YA, Sobolev Spaces. Springer-Verlag, Berlin, (1985). 
[30] M. MIRANDA, Superfici cartesiane generalizzate ed insiemi di perimetro localmente finito sui prodotti cartesiani, Ann. Scuola Norm. Sup. Pisa. 18(3) (1964), 515-542.

[31] Y. G. RESHETNYAK, Weak convergence of completely additive vector functions on a set, Siberian Math. J. 9 (1968), 1039-1045.

[32] W. RUDIN, Real and Complex Analysis. Mc Graw-Hill, New York, (1966).

[33] J. SERRIN, On the definition and properties of certain variational integrals, Trans. Amer. Math. Soc. 161 (1961), 139-167.

[34] A. I. VOL'PERT and S. I. HUDJAEV, Analysis in Classes of Discontinuous Functions and Equations of Mathematical Physics, Martinus Nijhoff Publishers, Dordrecht (1985).

Received and accepted 5 May 2005

To access this journal online:

http://www.birkhauser.ch 